\title{
Efficacy and safety of CD19 chimeric antigen receptor T cells in the treatment of 11 patients with relapsed/refractory B-cell lymphoma: a single-center study
}

\author{
Chen Huang ${ }^{1}$, Lili Wu ${ }^{1}$, Ruixia Liu ${ }^{1}$, Weijing Li $^{1}$, Zheng Li $^{1}$, Jianqiang $\mathrm{Li}^{2}$, Lihong Liu ${ }^{1}$, Baoen Shan ${ }^{3}$ \\ ${ }^{1}$ Department of Hematology, The Fourth Hospital of Hebei Medical University, Shijiazhuang, China; ${ }^{2}$ Hebei Senlang Biotechnology, Shijiazhuang, \\ China; ${ }^{3}$ Cancer Institute, The Fourth Hospital of Hebei Medical University, Shijiazhuang, China \\ Contributions: (I) Conception and design: L Liu, J Li; (II) Administrative support: B Shan; (III) Provision of study materials: C Huang, L Wu; (IV) \\ Collection and assembly of data: C Huang, R Liu; (V) Data analysis and interpretation: W Li, Z Li; (VI) Manuscript writing: All authors; (VII) Final \\ approval of manuscript: All authors. \\ Correspondence to: Baoen Shan; Lihong Liu. The Fourth Hospital of Hebei Medical University, Shijiazhuang, China. Email: baoenshan@hbydsy.com; \\ 13933838950@163.com.
}

Background: No effective treatment exist for patients with relapsed and refractory B-cell lymphoma, until the advent of anti-CD19 chimeric antigen receptor (CAR) T-cells. Therefore, this study aimed to explore the factors affecting the efficacy of anti-CD19 CAR T-cell and the adverse reactions of the therapy.

Methods: We recruited 11 patients with relapsed and refractory B-cell lymphoma. The number of antiCD19 CAR T-cells, proliferation, and adverse reactions were recorded in detail, to explore the relationship between the factors affecting the efficacy of anti-CD19 CAR T-cell and the long-term survival of patients.

Results: The 11 patients in our study had a total overall response rate of $100 \%$, after receiving anti-CD19 CAR T-cells. The median follow-up was 253 days (range, 130-1,017 days). The median overall survival (OS) and median progression-free survival (PFS) were not reached. After 3 months of treatment, the complete remission (CR) rate was 63.6\% (7/11). As of December 7, 2019, 5 patients had maintained CR for a period exceeding 1 year, including 2 patients who had maintained CR for more than 1,000 days. The patients who received 3 or 4 lines of chemotherapy were more likely to have sustained remission than the patients who received $<2$ or $>4$ lines of chemotherapy. Each of the 4 patients in the study who had diffuse large B cell lymphoma (DLBCL) progression all had high myc protein expression (positive incidence: $30-80 \%$ ). The incidence of Grade 2 cytokine release syndrome (CRS) was 36.4\% (4/11), and Grade 3 CAR T-cell-related encephalopathy syndrome (CRES) was experienced by 1 patient. The occurrence of adverse reactions was not significantly related to the infusion dose, peak amplification time, or maximum copy amount. The immunoglobulin levels of the four patients who died showed a significant downward trend. Interleukin$1 \beta$ (IL-1 $\beta$ ), interferon- $\gamma$ (IFN- $\gamma$ ), interleukin-10 (IL-10), and interleukin-17A (IL-17A) appeared to be associated with the occurrence of CRS and CRES.

Conclusions: Anti-CD19 CAR T-cell treatment is a new therapy for patients with relapsed and refractory B-cell lymphoma. Among the small sample size in this study, it demonstrated high efficiency and safety.

Trial registration: This trial is registered at clinicaltrials.gov (NCT01865617).

Keywords: B-cell lymphoma; efficacy; safety; chimeric antigen receptor T cells (CAR T cells)

Submitted Feb 20, 2020. Accepted for publication Jul 23, 2020.

doi: $10.21037 / \mathrm{atm}-20-4363$

View this article at: http://dx.doi.org/10.21037/atm-20-4363 


\section{Introduction}

B-cell lymphoma is a heterogeneous disease which comprises up to $66.3 \%$ of all lymphoma cases in China. Around half (50.2\%) of patients with B-cell lymphoma are diagnosed with diffuse large B cell lymphoma (DLBCL) (1). The majority of B-cell lymphomas are characterized by positive expression of CD20 (2), a membrane antigen which is associated with cell cycle initiation and differentiation.

Anti-CD20 monoclonal antibodies (such as rituximab) can have a significant impact in the treatment of B-cell lymphoma when combined with chemotherapy (3-5). Targeted therapy containing rituximab, in particular, can achieve a 5 -year overall survival (OS) rate of $75 \%$ in DLBCL patients (6). However, $30 \%$ to $40 \%$ of patients relapse, and in $10 \%$ of patients, resistance to initial and secondary drugs presents a challenge for effective treatment (7). These patients can expect a dire prognosis; the response rate to second-line chemotherapy in DLBCL patients is less than $30 \%$, dropping to $14 \%$ with third-line chemotherapy $(8,9)$. Even with high-dose chemotherapy combined with autologous hematopoietic stem cell transplantation (ASCT), the response rate is only $20 \%$ to $40 \%$ (10).

Lymphoma treatment has entered the era of immunotherapy. Chimeric antigen receptor (CAR) therapy is one of the promising tumor adoptive immune cell therapy methods developed in the past 20 years. CAR modified $T$ cells (CAR T cells) specifically recognize tumor cell surface antigens through the principle of antigen and antibody binding, to avoid endogenous $T$ cells from being restricted by the major histocompatibility complex, that is currently an effective treatment method to overcome hematological malignancies. Genetically engineered chimeric antigen receptor $T$ cells (CAR Ts) facilitate immunotherapy strategies with higher accuracy. CAR $\mathrm{T}$ allows $\mathrm{T}$ lymphocytes to express a specific CAR via genetic modification, through which the target antigens can be recognized and the target cells killed (11). CAR T cells have a strong affinity with specific tumor antigens and can efficiently kill tumor cells that express the corresponding antigen. CD19 is specifically expressed on the surface of B lymphocytes at different stages of differentiation. In normal tissues, CD19 is only expressed in mature B cells, precursor B cells, and plasma cells, but hematopoietic stem cells and non-hematopoietic stem cells do not express CD19. Most B cell malignant tumor cells express CD19, and CD19 antigen is expressed in more than $95 \%$ of B-cell lymphoma cases. As such, CD19-targeted CAR T cells can be used to treat B-cell lymphoma $(12,13)$.

A number of clinical studies have confirmed that anti-
CD19 CAR T-cells have a significant effect on relapsed/ refractory (R/R) B-cell lymphoma (14-16). Based on the remarkable efficacy of the ZUMA-1 study, the Food and Drug Administration (FDA) approved the first CAR T cell therapy for the treatment of specific types of NHL in 2017 $(17,18)$. Subsequently, in May 2018, the FDA approved the second indication of tisagenlecleucel (CTL019) for the treatment of adult patients with relapsed/refractory large B-cell lymphoma (19). The third product of CD19 CAR $T$ cell is lisocel (lisocabtagene maraleucel). The TRANSCEND study is based on CD19-targeted 4-1BB CAR $T$ cell therapy to treat relapsed/refractory and aggressive B-cell NHL. The above data suggest that CD19 CAR $T$ product is an effective rescue treatment for relapsed and refractory B-cell lymphoma (20). However, the adverse reactions experienced by patients during CAR $\mathrm{T}$ treatment needs to be addressed. Among these adverse reactions, cytokine release syndrome (CRS) and neurotoxicity are especially prominent. CRS refers to the release of a large number of cytokines after CAR $\mathrm{T}$ infusion which induces tissue damage and manifests as a syndrome, also known as a cytokine storm, which results in fever, tachycardia, and hypotension (21). In studies on CAR $\mathrm{T}$ for B-cell lymphoma, neurotoxicity has been reported in some patients, with side effects including confusion, tremors, ataxia, aphasia, lethargy, and seizures (22). This study set out to analyze the efficacy and adverse effects of CAR T-cell therapy in 12 patients with B-cell lymphoma who took part in clinical trials, with the aim of providing a theoretical basis for more reasonable and safe application of CAR T. We have obtained extremely high objective response rate (ORR). In addition to discussing the effectiveness and safety of CAR T treatment, we also observed the effects of immunochemotherapy on CAR $\mathrm{T}$ and long-term adverse reactions after CAR T treatment. This will give us a clue to the clinical practice of CAR T therapy.

We present the following article in accordance with the STROBE reporting checklist (available at http://dx.doi. org/10.21037/atm-20-4363).

\section{Methods}

\section{Patient selection}

The clinical data of 11 patients with relapsed and refractory B-cell lymphoma were collected. The study protocol was approved by the ethics committee of The Fourth Hospital of Hebei Medical University, and informed consent was obtained from all the patients. The study was conducted in accordance 


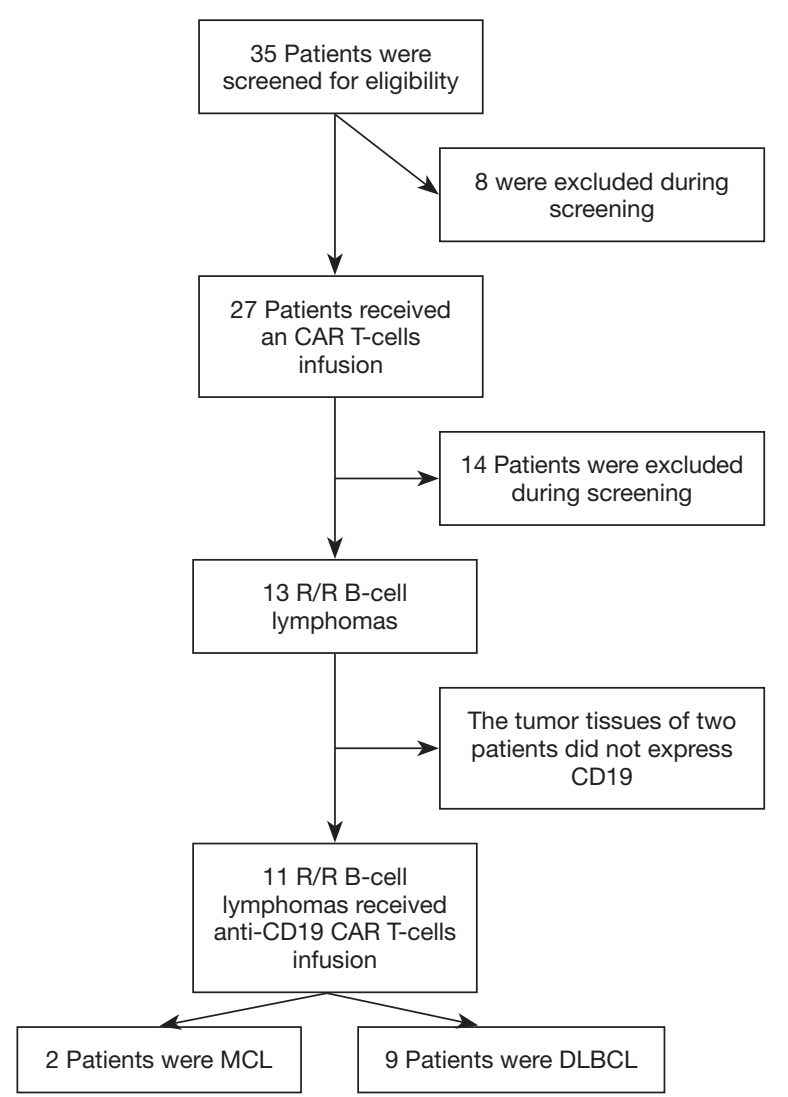

Figure 1 Screening, enrollment, and treatment. Among the 35 patients who were excluded from the study during screening, 8 patients were forced to withdraw due to rapid disease progression during CAR T-cell culture. Patients with unqualified histological subtypes were excluded, including 1 case with Hodgkin's lymphoma, 3 cases with multiple myeloma, and 2 cases with primary mediastinal DLBCL. Eight cases who had received with cd19CD22 double CAR-T cell therapy were also excluded. Two patients withdrew due to weak or no expression of CD19 in tumor tissue. CAR, chimeric antigen receptor; MCL, mantle cell lymphoma; DLBCL, diffuse large B cell lymphoma.

with the Declaration of Helsinki (as revised in 2013). This trial is registered at clinicaltrials.gov (NCT03121625).

The patients were diagnosed with DLBCL according to the 2008 World Health Organization classification criteria. CD19 expression in malignant tissue was confirmed by immunohistochemistry. The patients have no fertility plan since the diagnosis.

Patients with B-cell lymphoma that cannot achieve completely remedied by chemotherapy more than 2 cycles, or primary lymphoma that has not been remitted after first-line chemotherapy but is not suitable for intensive chemotherapy again. Exclusion criteria include left ventricular ejection fraction $<50 \%$; history of severe lung dysfunction; combined with other malignant tumors; patients with recurrence after allogeneic hematopoietic stem cell transplantation with grade 3 to 4 acute graft versus host disease (GvHD); active hepatitis.

The patients' complete clinical histories were gathered for analysis, and each patient underwent physical examination, including complete blood count, renal and liver function levels, serum albumin, lactate dehydrogenase (LDH), $\beta 2$ microglobulin, bone marrow biopsy, and computerized tomography scans of the brain, chest, abdomen, and pelvis. The patients were staged according to the Ann Arbor classification. Performance status was evaluated based on the ECOG scale, and risk stratification according to IPI and mIPI scores $(23,24)$. Previous treatment options and adverse events are detailed in Figure 1.

\section{Preparation of anti-CD19 CAR T cells and ex vivo assays}

The CAR19 gene was composed with an anti-CD19 scFv (FMC63), an IgG4 hinge, a CD28 transmembrane domain, a 4-1BB-derived costimulatory domain, and a CD3 $\zeta$ intracellular domain. The 1904B gene was composed with an anti-CD19 scFv (FMC63), a CD8 hinge and transmembrane domain, a 4-1BB-derived costimulatory domain, and a CD3 $\zeta$ intracellular domain. Both CAR19 and 1904B were combined with a truncated EGFR protein, which was linked by T2A sequence at the C-terminus of CAR. CAR T-cells were generated as previously described (25) (Figure S1). In brief, CD3 + T cells were isolated from the peripheral blood mononuclear cells by CliniMACS CD3 (Miltenyi Biotec). The $\mathrm{T}$ cells were activated by CD3/CD28 microbeads (Gibco), and cultured in TexMACS (Miltenyi Biotec) with $200 \mathrm{IU} / \mathrm{mL}$ of interleukin-2 (Miltenyi Biotec). Lentivirus-mediated CAR transduction was performed on the day 2 post-activation. The composition and purity of the CAR-T cells were assessed by flow cytometry. The CAR T-cells were harvested on day 14 (Figure 2).

\section{Treatment plan}

CAR T-cells were provided by Senlang Biotechnology. Each CAR T-cell came with a quality report, in compliance with good manufacturing practice. The chemotherapy treatment comprised fludarabine $25 \mathrm{mg} / \mathrm{m}^{2} / \mathrm{d}(\mathrm{d} 1-3)$ and cyclophosphamide $900 \mathrm{mg} / \mathrm{m}^{2} / \mathrm{d}(\mathrm{d} 3-4)$. A single infusion of anti-CD19 CAR T-cells was administered on second day 


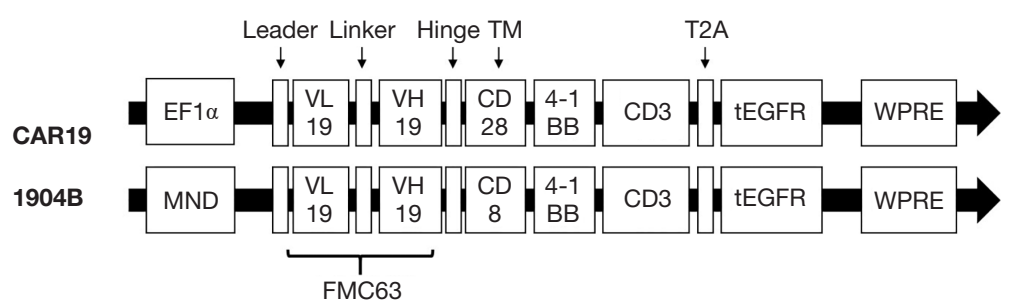

Figure 2 Preparation of Anti-CD19 CAR T Cells. The CAR19 gene was composed with an anti-CD19 scFv (FMC63), an IgG4 hinge, a CD28 transmembrane domain, a 4-1BB-derived costimulatory domain, and a CD3 $\zeta$ intracellular domain. The 1904B gene was composed with an anti-CD19 scFv (FMC63), a CD8 hinge and transmembrane domain, a 4-1BB-derived costimulatory domain, and a CD3 $\zeta$ intracellular domain. Both CAR19 and 1904B were combined with a truncated EGFR protein, which was linked by T2A sequence at the C-terminus of CAR. CAR-T cells were generated as previously described. In brief, CD3+ T cells were isolated from the peripheral blood mononuclear cells by CliniMACS CD3 (Miltenyi Biotec). T cells were activated with CD3/CD28 microbeads (Gibco), and cultured in TexMACS (Miltenyi Biotec) with $200 \mathrm{IU} / \mathrm{mL}$ of IL-2 (Miltenyi Biotec). Lentivirus-mediated CAR transduction was performed on day 2 post-activation. The composition and purity of the CAR-T cells were assessed by flow cytometry. The CAR-T cells were harvested on day 14. CAR, chimeric antigen receptor; IL-2, interleukin-2.

after chemotherapy. Promethazine was injected to prevent an allergic occurring before the transfusion. The patients in this study did not receive exogenous interleukin-6 (IL-6). The cells dose ranged from 1.8 to $3 \times 10^{6}$ CAR-positive T-cells/kg during the study. Peripheral blood cells were cultured for 14 days in the laboratory and then returned to the patient. The patients' physical condition was closely monitored after the transfusion, and peripheral blood was extracted at days $4,7,10,14,21$, and 28 post-transfusion, and CAR T-cell proliferation and cytokine levels in the patients were detected.

\section{Assessing efficacy and safety}

The efficacy of the treatment was judged using the following categories based on the the National Comprehensive Cancer Network 2019 guidelines for nonHodgkin's lymphoma: complete remission (CR), partial response (PR), stable disease (SD), and progressive disease (PD). CRS was classified according to the revised CRS classification system proposed by Lee et al. (26), while cellrelated encephalopathy syndrome (CRES) classification was based on the management of toxicities suggested by Neelapu et al. (27).

\section{Statistical analysis}

The number of cases involved in this study was relatively small and, as such, mainly descriptive analysis was adopted. The numerical variables are represented by maximum, minimum, and median. Categorical variables are shown in tables. A survival curve was constructed using the KaplanMeier method with Rothman 95\% confidence intervals (CIs). All statistical analyses were performed with SPSS version 17.0 software (SPSS Inc., USA). OS was defined as the time between the date of infusion and the date of death. PFS was defined as the time elapsed between the date of infusion and the date of progression, relapse, or death.

\section{Results}

\section{Clinical characteristics of patients before CAT therapy}

The clinical characteristics of the 11 patients enrolled in this study are listed in Table 1 . The patients had a median age of 49 (range, 29-69) years, and 36.4\% (4/11) of the patients were $>60$ years old. Males accounted for $72.7 \%(8 / 11)$ of the patients. The majority of the patients $(81.8 \%, 9 / 11)$ presented with advanced-stage (III/IV) disease. Extranodal involvement was observed in $45.5 \%$ $(5 / 11)$ of the patients, in areas including the bone marrow, skin, breasts, gastrointestinal tract, and central nervous system. Most of the patients had multiple extranodal organ involvement. Pathological types included 2 cases of relapsed and refractory mantle cell lymphoma, and 9 cases of relapsed and refractory DLBCL. High Ki67 ( $\geq 70 \%)$ accounted for $72.7 \%(8 / 11)$ of the patients. Five cases of DLBCL originated from activated B-cells, two of which were double-expression lymphomas. All of the patients had received multi-course and multi-line chemotherapy, with 
Table 1 Patient clinical characteristics before CAT therapy

\begin{tabular}{|c|c|}
\hline Characteristic & Number (no.) of patients \\
\hline \multicolumn{2}{|l|}{ Age (years) } \\
\hline$>60$ & $4 / 11(36.4 \%)$ \\
\hline \multicolumn{2}{|l|}{ Sex } \\
\hline Male & $8 / 11(72.7 \%)$ \\
\hline Extranodal involvement & $5 / 11(45.5 \%)$ \\
\hline \multicolumn{2}{|l|}{ Pathological type } \\
\hline $\mathrm{MCL}$ & $2 / 11(18.2 \%)$ \\
\hline DLBCL & $9 / 11(81.8 \%)$ \\
\hline Germinal center B-cell type & $4 / 9(44.4 \%)$ \\
\hline Double expressing & $2 / 9(22.2 \%)$ \\
\hline High Ki67 (positive $\geq 70 \%$ ) & $8 / 11(72.7 \%)$ \\
\hline No. of previous courses of antineoplastic therapy & Median 12 (range, 8-34) \\
\hline No. of previous lines of antineoplastic therapy & Median 3 (range, 2-6) \\
\hline Large mass at first diagnosis (major axis $>7 \mathrm{~cm}$ ) & $5 / 11(45.5 \%)$ \\
\hline Large mass before infusion of anti-CD19 CAR-T cells (major axis $>7 \mathrm{~cm}$ ) & $4 / 11(36.4 \%)$ \\
\hline
\end{tabular}

MCL, mantle cell lymphoma; DLBCL, diffuse large B-cell lymphoma; CAR, chimeric antigen receptor. Double expressing, MYC $\geq 40 \%$ positive, BCL2 $\geq 50 \%$ positive.

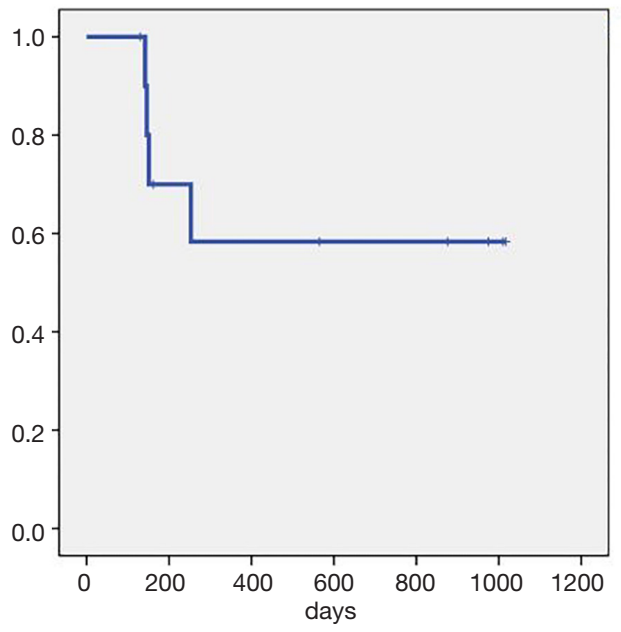

Figure 3 Survival curve for all patients. a median 3 (range, 2-6) lines and a median course of 12 (range, 8-34).

\section{Patients with sustained remission}

Overall, the patients had a median follow-up time of 253 days (130-1,017 days). The ORR (CR + PR) rate was $100 \%$. The median OS and median PFS were not reached. The CR rate after 1 month was $27.3 \%$ (3/11). The CR rate after 3 months was $63.6 \%(7 / 11)$, and the PD rate was $36.4 \%$ (4/11). As of December 7, 2019, there were 5 patients who had achieved CR lasting over 1 year, including two patients for whom CR had lasted over 1,000 days. By the same point in time, four patients had died: two died from disease progression, one died after acquiring a severe lung infection, and the other died from gastrointestinal bleeding unrelated to the primary disease (Figure 3, Table 2).

In this study, the case of Patient No. P040 was particularly remarkable. She underwent treatment with 


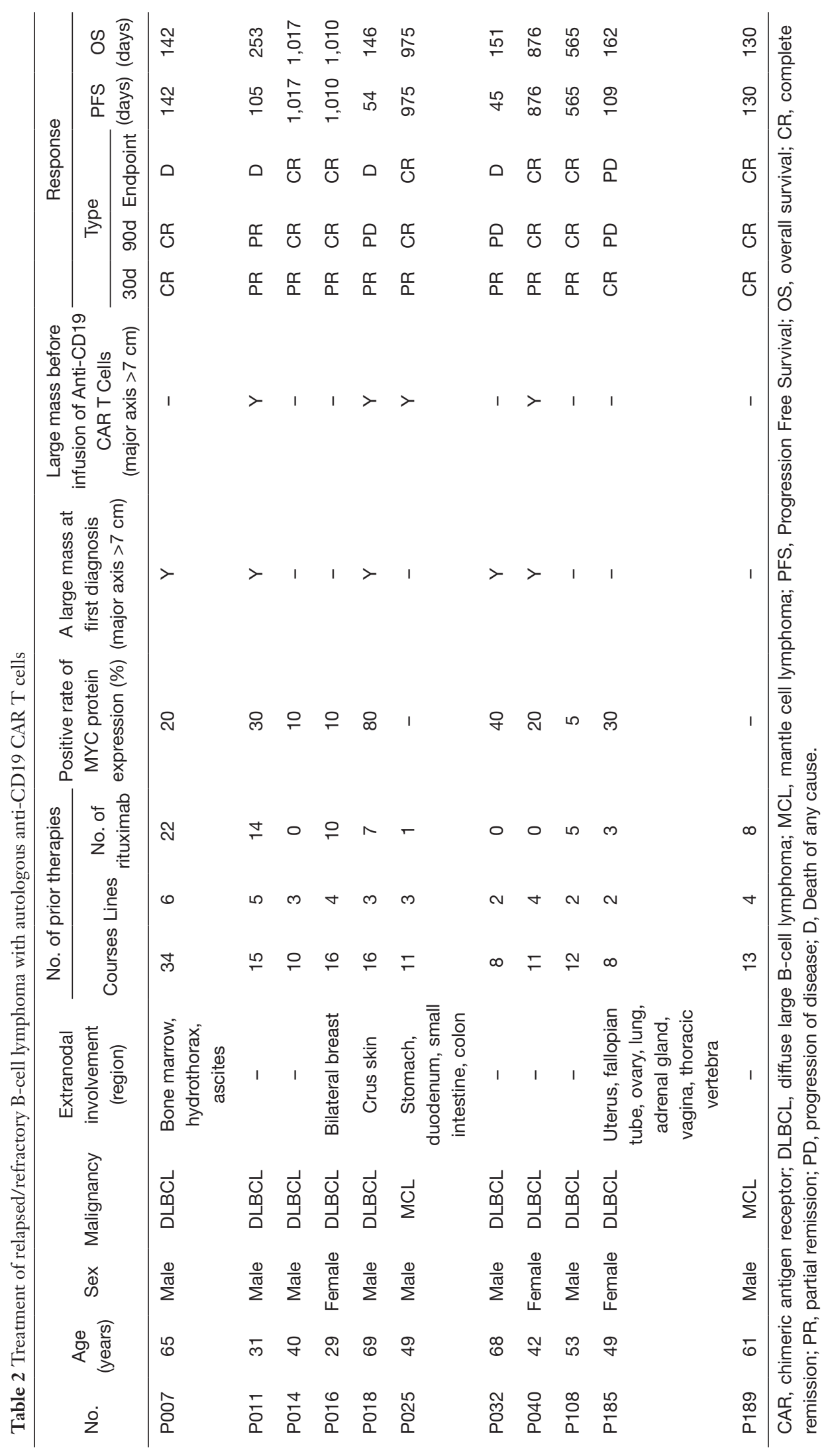



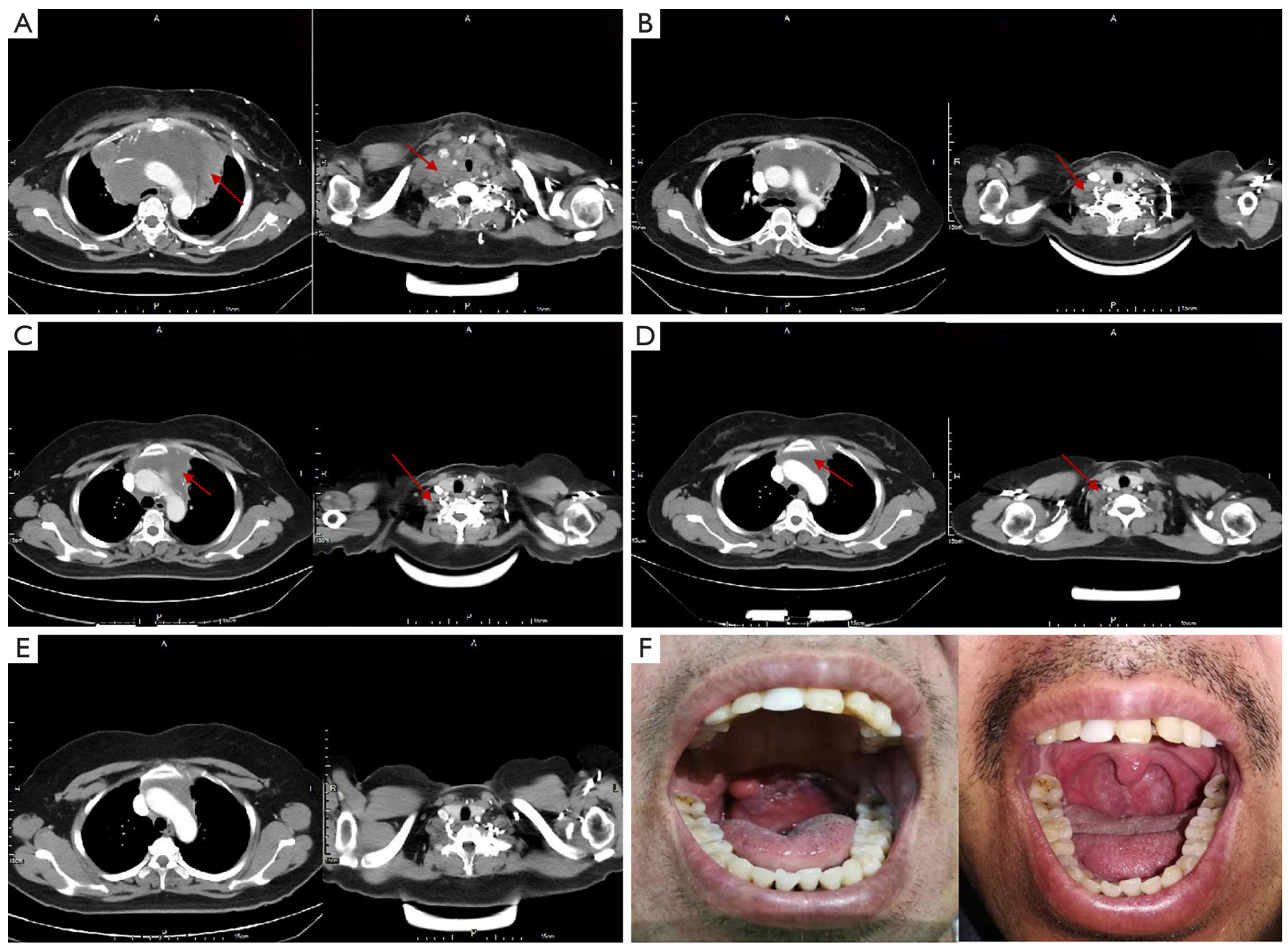

Figure 4 Changes in lesions of patients No. P040 and P189 before and after CAR-T cell treatment. A neck and chest CT scan of Patient No. P040 showed a huge mass in the mediastinum and a lymphadenectasis in the right supraclavicular fossa on day 0 (A). Imaging at day 28 (B), day 90 (C), and day 180 (D) showed that the mass continued to shrink to complete remission until day 720 (E). Patient No. P189 had a large mass on the tonsil (F. left). After treatment on the CD19 CAR protocol, he entered a CR that was ongoing after 130 days (F. right). CAR, chimeric antigen receptor; CR, complete remission.

eight cycles of cyclophosphamide, doxorubicin, vincristine, and prednisone (CHOP), which resulted in a CR that lasted 2 months before relapse. Subsequently, she received one cycle of dexamethasone ifosfamide, carboplatin, and etoposide (DICE) chemotherapy; the result was PD. $\mathrm{PD}$ continued after the replacement of her regimen with gemcitabine, cisplatin, and dexamethasone (GDP). Finally, she was administered a regimen of etoposide, methylprednisolone, cisplatin, and cytarabine (ESHAP), and PD was maintained. During her treatment, the patient experienced severe dyspnea, hoarseness, and difficulty laying down due to superior vena cava obstruction caused by the compression of the large mediastinal mass. She was treated according to the CD19 CAR protocol and significant shrinkage of the mass was observed in the short term. Fortunately, she entered a CR that was ongoing after 876 days (Figure $4 A, B, C, D, E$ ).

Another notable patient was No. P189, who was diagnosed with mantle cell lymphoma. He received four different treatment regimens, including rituximab, ibrutinib, and lenalidomide, before enrolling onto the CD19 CAR protocol. Progression of disease after his last salvage chemotherapy regimen. The patient suffered severe dysphagia and dyspnea due to a large pharyngeal mass. After receiving treatment according to the CD19 CAR protocol, he entered a CR that was ongoing after 130 days (Figure 4F). 
A

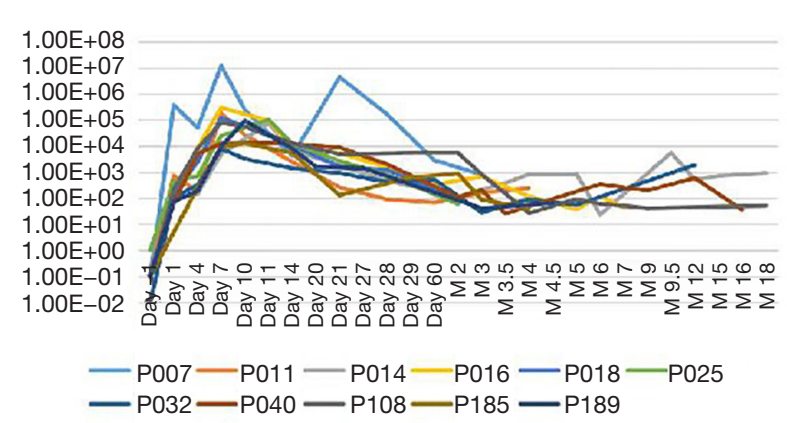

C $\triangle$ The dose of CAR-T $\triangle$ Maximum copy of DNA $\triangle$ CRS level $\triangle$ CRES level

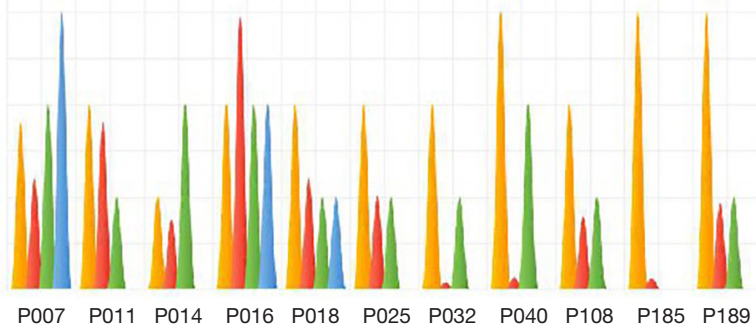

B Temperature

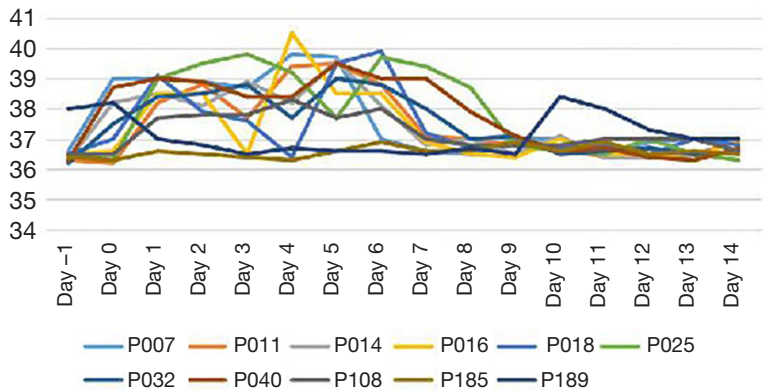

D

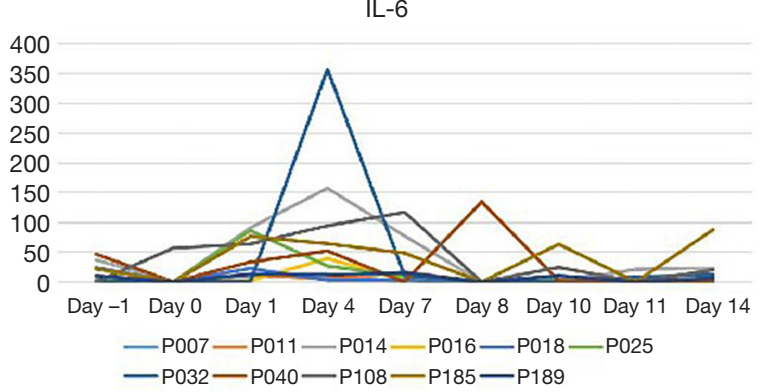

Figure 5 Comparison of the differences in temperature, dose and copy number of anti-CD19 CAR T-cells, adverse reactions, and IL-6 levels. Temperature changes in all of the patients on day 0 to day 14 (A). QPCR changes in all of the patients on day 0 to 18 months (M18). (B) Comparison of the differences among infusion dose of CAR-T cells $\left(10^{6} / \mathrm{kg}\right)$, CRS level, CRES level and maximum DNA copy amount $\left(10^{5}\right)$ of all the patients (C). Comparison between IL-6 levels in all patients (D). CAR, chimeric antigen receptor; QPCR, quantitative real-timePCR; IL-6, interleukin-6.

\section{Relationship between dose and copy number of anti-CD19 CAR T-cells and adverse reactions and prognosis}

There was no significant correlation observed between infusion dose, peak amplification time, and maximum copy volume. In the 8 weeks after the infusion, 10 out of 11 patients $(90.9 \%)$ developed CRS of any grade. The incidences of grade 2 and grade 1 CRS were $36.4 \%$ (4/11), and $54.5 \%(6 / 11)$, respectively, while 1 patient did not experience any CRS. The most common grade 1 CRS symptom was fever, $90.9 \%$ (10/11) (Figure 5A). With Grade 2 CRS, pulmonary infection and hypotension were experienced by $27.3 \%(3 / 11)$ and $9.1 \%$ (1/11) of the patients, respectively (Table 3). CRES occurred in 3 patients: 1 case showed grade 3 convulsion and confusion, 1 case showed grade 2 dysphoria and delirium, and 1 case showed grade 1 confusion and delayed response. All 3 patients had shorter peak amplification times (7 days) and higher maximum copy value $\left(1 \times 10^{5}\right)$. CD19 CAR T-cell deoxyribonucleic acid was detected by quantitative real-time-PCR (QPCR) in all patients, which showed the peak of CAR T amplification was concentrated on d7-11 (Figure 5B). Patient No. P007, who had the largest copies $\left(2.95 \times 10^{5}\right)$ displayed the most severe CRS and CRES (Figure 5C). It is worth mentioning that this patient underwent the most courses of chemotherapy, the most lines of treatment, and had a shorter survival time. The changes of IL-6 in the peripheral blood of the 11 patients are shown in Figure 5D. Patient No. P016 acquired grade 2 CRS and CRES, while no CRS or CRES occurred with Patient No. P185. The number of cytokines, including IL-1, interferon- $\gamma$ (IFN- $\gamma)$, monocyte chemoattractant protein-1 (McP-1), interleukin-10 (IL-10), and interleukin17A (IL-17A), of these 2 patients were compared (Figure $6 A, B, C, D, E)$. The results revealed that IL- $1 \beta$, IFN- $\gamma$, IL-10, and IL-17A appeared to be associated with the occurrence of CRS and CRES. Patient No. P016 had higher levels of IL$1 \beta, \mathrm{IFN}-\gamma$, and IL-17A, and lower IL-10 levels than Patient 
Table 3 Adverse reactions and cytokine of levels after treatment

\begin{tabular}{|c|c|c|c|c|c|c|c|}
\hline $\begin{array}{l}\text { Patient } \\
\text { No. }\end{array}$ & $\begin{array}{c}\text { Highest } \\
\text { temperature } \\
\left({ }^{\circ} \mathrm{C}\right)\end{array}$ & $\begin{array}{c}\text { Fever } \\
\text { duration } \\
\text { (d) }\end{array}$ & $\begin{array}{l}\text { Maximum copy } \\
\text { amount }\left(\times 10^{5}\right)\end{array}$ & $\begin{array}{c}\text { Peak } \\
\text { amplification } \\
\text { time (d) }\end{array}$ & $\begin{array}{c}\text { No. of CAR } \\
\text { positive T Cells } \\
\text { Infused }\left(\times 10^{6} / \mathrm{kg}\right)\end{array}$ & \multicolumn{2}{|c|}{ Toxicities } \\
\hline P011 & 39.5 & 6 & 1.805 & 7 & 2.0 & 1 (fever, sore muscles) & 0 \\
\hline P016 & 38.5 & 6 & 2.950 & 7 & 2.0 & 2 (fever, weak, loss of appetite) & 2 (dysphoria, delirium) \\
\hline P018 & 39.9 & 3 & 1.200 & 7 & 2.0 & 1 (fever) & $\begin{array}{c}1 \text { (confusion, delays in } \\
\text { response) }\end{array}$ \\
\hline P025 & 39.8 & 8 & 1.014 & 11 & 2.0 & 1 (fever) & 0 \\
\hline P108 & 38.3 & 6 & 0.783 & 7 & 2.0 & 1 (fever) & 0 \\
\hline P185 & 37.1 & 0 & 0.127 & 10 & 3.0 & 0 & 0 \\
\hline P189 & 37.9 & 3 & 0.935 & 10 & 3.0 & 1 (fever) & 0 \\
\hline
\end{tabular}

CAR, chimeric antigen receptor; CRS, cytokine release syndrome; CRES, CART-cell-related encephalopathy syndrome.

No. P185 (Figure 6A,B,C,D). The immunoglobulin levels of the four patients who died showed a significant downward trend (Figure 6F).

One of the patients who died, Patient No. P007, who was diagnosed with DLBCL, received six different treatment regimens, including rituximab, bendamustine, and lenalidomide, before being treated according to the CD19 CAR protocol. After treatment based on the CD19 CAR protocol, he entered a CR that lasted 142 days. Patients in the remission period have persistently declining hypogammaglobulinemia and, as a result, recurring lung infections can occur, including infections mixed with aspergillus, cytomegalovirus, and bacteria. In this case, the patient died of uncontrollable lung infection which led to respiratory failure.

\section{Discussion}

B-cell lymphoma is the most common subtype of nonHodgkin's lymphoma. In recent years, the application of anti-CD20 antibody in combination with treatment methods including chemotherapy, radiotherapy, and ASCT, has seen the survival time of newly treated patients significantly extended. Nevertheless, some B-cell lymphomas are still difficult to cure.
For some patients with DLBCL, the conventional treatment regimen is not effective, and they experience no response to the RCHOP regimen (rituximab, cyclophosphamide, pyrandoxorubicin, vincristine and prednisone acetate); the result is primary refractory DLBCL. About $40 \%$ of DLBCL patients who are sensitive to first-line treatment relapse within 5 years (28). For these DLBCL patients, the standard second-line treatment is salvage chemotherapy + ASCT $(29,30)$, but secondline treatment has been reported to only benefit $23-29 \%$ of primary refractory DLBCL cases (31), and the PFS is only 3 months (9). Patients who relapse after second-line treatment have a median OS of only 10 months $(32,33)$.

The refractory or relapse of $\mathrm{B}$-cell lymphoma is a multifactorial process, and multiple mechanisms are involved. The best curative effect is possible through individualized comprehensive treatment. Therefore, there is still a lack of effective treatment for relapsed/ refractory B-cell lymphoma, and new treatment technologies to improve the prognosis of these patients are desperately needed. Indeed, the emergence of CD19 CAR T treatment may open up such a treatment option for patients with relapsed/ refractory B-cell lymphoma.

CAR is an artificial fusion protein composed of intracellular signal transduction region, transmembrane 

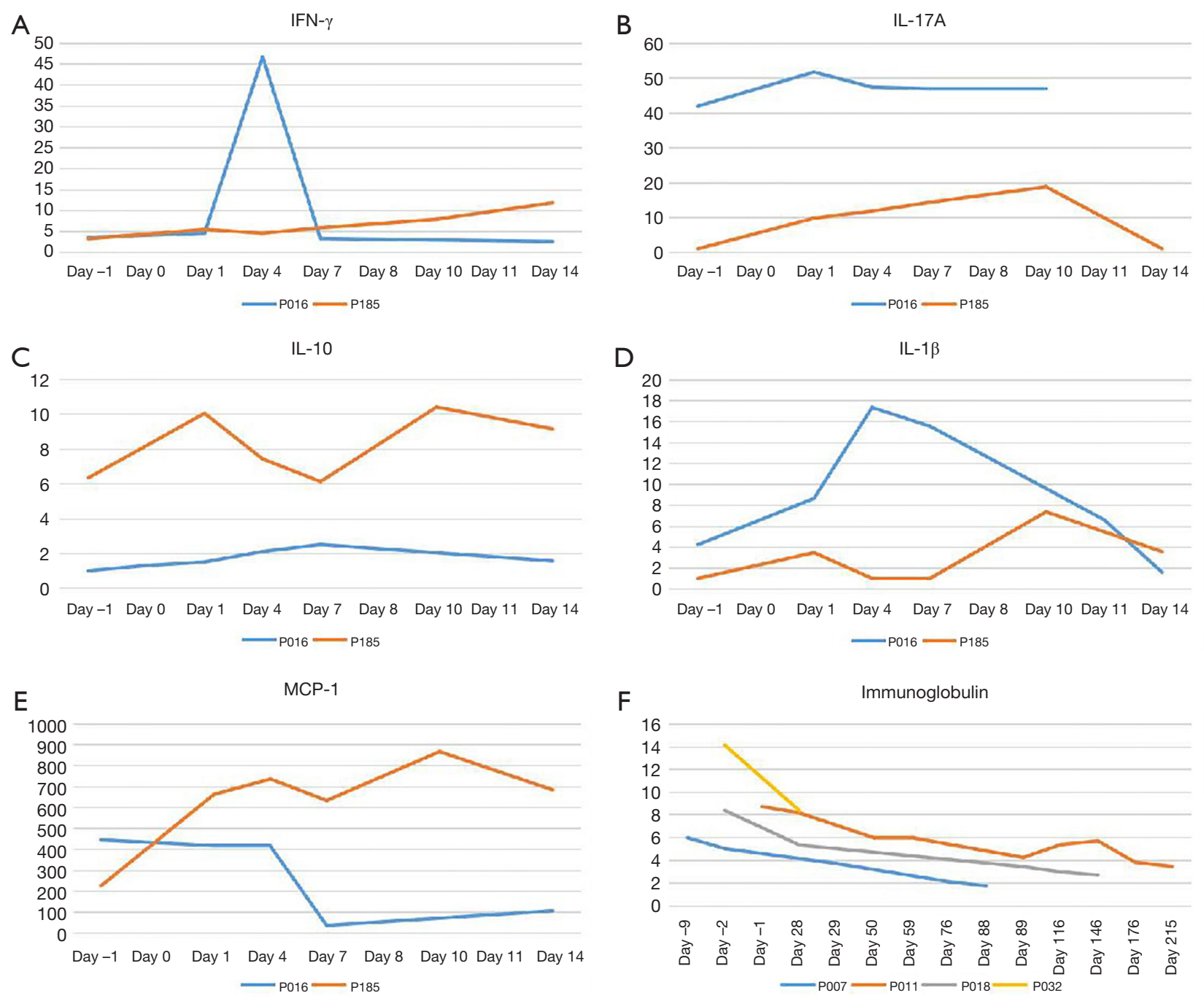

Figure 6 Comparison of the differences in cytokines and immunoglobulins. Comparison of the differences in IFN- $\gamma$ (A), IL-17A (B), IL10 (C), IL-1 $\beta$ (D), and McP-1 (E) between Patients Nos. P016 and P185. The immunoglobulins of the four patients who died showed a significant downward trend (F). IFN- $\gamma$, interferon- $\gamma$; IL-17A, interleukin-17A; IL-10, interleukin-10; IL-1 $\beta$, interleukin-1 $\beta$.

region, and extracellular antigen-binding region. Surface chimeric CAR protein $\mathrm{T}$ cells are known as CAR T (34). CD19 expression occurs throughout the entire B-cell development process, and CD19 is therefore an ideal target for CAR-T treatment of B-cell lymphoma. CAR $T$ targeting of CD19 is the most widely used and comprehensively studied adoptive cellular immunotherapy in clinical practice (35). With an increasing amount of indepth research of CAR T that specifically recognizes CD19 on B-cell surfaces, its effectiveness in patients with relapsed/ refractory B-cell lymphoma has gradually improved. In addition to CD19, CD20 (36) and CD22 (37) are potential targets.

While CAR T technology has shown great potential for treating B-cell lymphoma, the adverse reactions it can entail still present a problem that cannot be ignored, and serious adverse reactions are the biggest obstacle limiting its efficacy. Common problems during CAR T treatment include CRS, CRES, tumor lysis syndrome, and off-target effects. This study explored the effectiveness and safety of CD19 CAR T in relapsed/refractory B-cell lymphoma based on a small sample of cases. The patients in this study demonstrated a high ORR rate, similar to the $81 \%$ and the $77.3 \%$ response rates reported by Rosenberg and Xiao, 
respectively (38). However, with the rapid remission of tumors, the degree of CAR T-cell replication also rapidly decreases. Due to the escape mechanism of tumor cells, cellular immunotherapy still cannot achieve long-lasting effects and disease recurrence is common.

The traditional prognostic model for DLBCL suggests that mass size is an independent factor of poor prognosis. All patients with large masses should receive radiotherapy in the affected area (39). In our study, 5 patients with large masses (major axis of lumps $>7 \mathrm{~cm}$ ) at the time of initial diagnosis were all DLBCL. The large masses in these patients all indicated poor prognosis and disease progression in the short term, and despite undergoing CD19 CAR T-cell treatment, they still had a poor median OS of 151 days (142-876 days). In contrast, the patients without large masses achieved good response rates and OS. Meanwhile, those patients with large masses (diameter $\geq 7.5 \mathrm{~cm}$ ) prior to the commencement of CD19 CAR $\mathrm{T}$ treatment had good prognosis despite not receiving radiotherapy after treatment. This suggests that CD19 CAR $T$ treatment cannot overcome the poor prognosis of DLBCL related to large mass size at initial diagnosis, but the prognosis of patients with large masses that appear only after relapse is not negatively affected. It is possible that a tumor with a large mass at the time of initial diagnosis may have a more complicated local microenvironment. Although a large mass formed after relapse is resistant to conventional chemotherapy, the cell component may be more unitary. Further study involving large sample sizes and long-term follow-up are required to confirm this finding.

Discovered in 1979, the MYC gene is a proto-oncogene which encodes protein and can be used as a transcription factor to participate in cell cycle regulation, proliferation, apoptosis, energy synthesis, and metabolism (40-42). The MYC gene is an important diagnostic indicator of DLBCL, based on the WHO classification of lymphoid tumor tissue in 2016 (43). Research has shown that an increase in MYC gene copy number and amplification is a factor of poor prognosis in DLBCL $(44,45)$. MYC protein expression is also indicative of a worse prognosis (46). The four patients with DLBCL progression in this study all had high MYC protein expression (percentage range, 30-80\%), and included 2 cases of double-expression lymphoma with 90\% Ki67 positivity. MYC protein expression in the other patients who experienced good curative effect was less than $30 \%$. CD19-positive cells were not detected in the relapsed tissue biopsy of 2 patients.

It has been suggested that CD19 CAR T treatment clears
CD19-positive cells in patients, but does not clear CD19negative or weakly expressed tumor cells, which offers a possible immune escape mechanism for these tumor cells (47-49). The continued activation of the MYC pathway and the involvement of other molecular pathways in the tumor cells gradually formed dominant clones, leading to recurrence and disease progression in these patients. Extensive involvement of multiple parts and extranodal invasion are all poor prognostic factors of aggressive B-cell lymphoma (50).

Regardless of the adjustment of chemotherapy regimen and increase of chemotherapy dose before the introduction of rituximab, or targeted combination therapy in the era of rituximab, and various new drug treatments, such as lenalidomide and ibtinib, the unfavorable prognosis caused by extensive disease and extranodal infiltration with B-cell lymphoma has not been reversed. Our small-sample study found that the effect of CD19 CAR T treatment was not affected by extensive involvement of the disease and extranodal infiltration, except with testicular lesions. There was no obvious correlation stage, which may be related to the fact that the mechanism of cellular immunotherapy is completely different from traditional treatment. Therefore, traditional prognostic factors may not be suitable for patients who undergo cellular immunotherapy. Three patients in our study had never used rituximab; these had a lower maximum copy volume. The 2 patients who had previously received more than 10 doses of rituximab had a higher maximum copy volume than the other patients. The maximum copy amount of DNA was not related to the number of CD19 CAR T-cells transfused, nor was it related to OS. The patients who had received more chemotherapy cycles and rituximab progressed to a more refractory disease state and had a worse prognosis. Two patients who had previously received more than 10 doses of rituximab had undergone 5 and 6 lines of treatments, respectively. After CD19 CAR T treatment, they still relapsed and progressed in a short period of time.

It is debatable whether patients can benefit from earlier intervention with CD19 CAR T treatment. It is also unclear whether rituximab, as an established first-line treatment, is synergistic with CD19 CAR T treatment. Due to the effectiveness of the previous CD19 CAR T treatment and the controllability of adverse reactions, we tried to enable patients who had high-risk initial diagnosis, refractory relapse, and initial drug resistance to undergo earlier CD19 CAR T therapy, after failure of second-line chemotherapy, but the effect did not meet our expectations. Three patients 
underwent fewer treatment courses and less rituximab treatment, two of whom had disease recurrence in the short term. The other patient, who received more courses and more rituximab, was still in remission so far with a good prognosis. Although some patients develop chemotherapy resistance, multiple courses of rituximab or the addition of new drugs may have potential long-term effects on molecular changes in patients with refractory DLBCL $(51,52)$. However, patients who undergo CAR T treatment have longterm immunosuppression afterwards, due to therapy with monoclonal antibody. Sustained low-level humoral immunity in patients who have severe hypogammaglobulinemia can make them prone to severe infections. In our study, the patients who achieved continuous CR were generally those who had undergone 3 or 4 lines chemotherapy. They had more courses and more adequate application of rituximab. Therefore, we recommend that patients undergo CD19 CAR $\mathrm{T}$ as soon as possible after more adequate treatment. We cannot ignore the role of targeted drug therapy and other new drug therapies, but how to combine them needs further exploration. Furthermore, we must also pay more attention to the risk of fatal infection in patients during long-term remission.

The two patients with the highest QPCR peaks in our study, P007 and P016, had relatively severe CRS and CRES. The occurrence time of CRS and CRES coincided with the peak time of QPCR, suggesting a significant correlation existed between the replication level of CD19 CAR T-cells and the occurrence of serious adverse reactions. Therefore, controlling the number of CD19 CAR T-cells is a feasible method to reduce serious adverse reactions.

Multiple analyses have suggested that IL- 6 may be a central regulator of CRS in CAR T treatment (53-55). With the successful use of IL-6 receptor inhibitors in acute lymphoblastic leukemia patients with deadly CRS, the inhibition of the IL-6 pathway is widely used to treat CRS (55). In many cases of severe CRS, the use of the IL-6 receptor inhibitor tocilizumab quickly relieved symptoms and did not clear the CAR T-cells or significantly reduce the effect of the treatment. Its effect on the duration of CR calls for further study. Our research did not show a significant association between IL-6 and CRS, and we did not use IL-6 receptor inhibitors as part of our treatment.

Patients Nos. P007, P016, and P018 acquired CRS and CRES, and endured a small dose of dexamethasone $(5 \mathrm{mg})$, achieving a positive effect. QPCR showed a slow decline in CD19 CAR T-cell replication, but in Patient No. P007, cell replication increased repeatedly after the decline. As the
CD19 CAR T-cell increased again, there was no recurrence of CRS. Aside from CD19 CAR T-cell replication level, there may be other regulatory mechanisms involved in the occurrence of CRS. Adverse reactions to treatment are an important influencing factor that limited CD19 CAR $\mathrm{T}$ treatment $(14,56,57)$. There were no treatment-related deaths during our research. CRES did occur in neither of the 2 patients who were enrolled after we improved the molecular structure of CAR. Among these, only 1 patient had Grade 1 CRS, which was not significantly related to the infusion dose, peak amplification time, and maximum copy amount, although the other experienced short-term recurrence and progression.

We successfully performed low-toxicity CD19 CAR $\mathrm{T}$ treatment by changing CAR molecules. CAR19 differs from 1904B in two aspects in the lentiviral vector sequence. Firstly, the structure of CAR molecules is different: CAR19 uses the IgG4 hinge region and the transmembrane region of CD28, while 1904B uses the CD8 hinge and the transmembrane region. Secondly, CAR molecules express a different promoter: CAR19 transcribes the CAR molecule through the EF1a promoter, while 1904B uses the MND promoter. We also compared two CAR T-cells that used different promoters to express the same CAR structure. In this unpublished result, it was shown that 1904B CAR T-cells can effectively kill tumors when co-cultured with tumor cells, but fewer cytokines are produced. We predict it will become a secure product for treatment of B-cell lymphoma. In comparative clinical trials, it was also found that the subjects who returned to $1904 \mathrm{~B}$ experienced a more moderate rise in temperature. For example, Patients Nos. P185 and P189 did not develop significant CRS and CRES. Even fever, as the most common symptom, did not occur (Figure 5A), but No. P185 showed PD on $\mathrm{d} 109$, and No. P186 continued with CR until the endpoint of the followup period. Therefore, the effectiveness of such changes needs to be further confirmed by long-term follow-up of large sample cases.

In addition to the adverse reactions in the course of treatment, we also observed serious adverse reactions caused by hypogammaglobulin. Almost all of the cases had hypogammaglobulinemia, and the decrease in globulin was particularly obvious in the four cases who died. Patients Nos. P007 and P011 died of respiratory failure caused by lung infection in the remission period of CR. We believe that severe infections caused by hypogammaglobulin after CAR $T$ treatment may result in long-term adverse reactions that affect prognosis. Therefore, immunoglobulin levels 
need to be monitored more frequently and over the longterm, especially in the rituximab era, when the duration and severity of hypogammaglobulinemia may be more severe.

\section{Conclusions}

In conclusion, our small sample report suggests that this new cell therapy provides an effective rescue method for patients with relapsed and refractory B-cell lymphoma. This treatment may require heightened accuracy in terms of the selection of suitable patients. Which B-cell lymphoma patients are suitable for CD19 CAR T treatment needs further exploration? At the same time, how to distinguish these high relapse patients and give sequential stem cell transplantation and how to integrate targeted drugs, new drugs, and other treatment methods still require further investigation. Manufacturing multitarget products of CAR $\mathrm{T}$ and reducing the adverse effects are our ongoing research goals. Severe adverse effects, in particular, will limit the application of CAR T technology. By making technical improvements, we have produced CAR T products with very low adverse reactions. Preliminary clinical trials have also demonstrated higher efficacy and lower toxicity. However, the long manufacturing time still limits the application of CAR T products. For patients with short-term recurrence, a universal CAR $T$ product desperately needs to be developed to improve the efficiency of CAR T therapy and save costs. This study is based only on a very small sample of case reports. The serious shortage of cases makes our study limited, and so large-scale studies are called for to verify our findings; at the same time, the long-term efficacy of the CAR $T$ treatment needs to be further studied.

\section{Acknowledgments}

Funding: None.

\section{Footnote}

Reporting Checklist: The authors have completed the STROBE reporting checklist. Available at http://dx.doi. org/10.21037/atm-20-4363

Data Sharing Statement: Available at http://dx.doi. org/10.21037/atm-20-4363

Conflicts of Interest: All authors have completed the ICMJE uniform disclosure form (available at http://dx.doi. org/10.21037/atm-20-4363). The authors have no conflicts of interest to declare.

Ethical Statement: The authors are accountable for all aspects of the work in ensuring that questions related to the accuracy or integrity of any part of the work are appropriately investigated and resolved. The study was conducted in accordance with the Declaration of Helsinki (as revised in 2013). The study protocol was approved by the ethics committee of The Fourth Hospital of Hebei Medical University (No. 2016040), and informed consent was obtained from all the patients.

Open Access Statement: This is an Open Access article distributed in accordance with the Creative Commons Attribution-NonCommercial-NoDerivs 4.0 International License (CC BY-NC-ND 4.0), which permits the noncommercial replication and distribution of the article with the strict proviso that no changes or edits are made and the original work is properly cited (including links to both the formal publication through the relevant DOI and the license). See: https://creativecommons.org/licenses/by-nc-nd/4.0/.

\section{References}

1. Xu W, Fan L, Miao Y, et al. Distribution pattern of lymphoma subtypes in China: a nationwide multicenter study of 10002 cases. Journal of Diagnostics Concepts \& Practice 2012;11:111-15.

2. Cheson BD, Leonard JP. Monoclonal antibody therapy for B-cell non-Hodgkin's lymphoma. N Engl J Med 2008;359:613-26.

3. Coiffier B, Haioun C, Ketterer N, et al. Rituximab (antiCD20 monoclonal antibody) for the treatment of patients with relapsing or refractory aggressive lymphoma: a multicenter phase II study. Blood 1998;92:1927-32.

4. Fan X, Zhong HJ, Zhao BB, et al. Metformin prolonged the survival of diffuse large B-cell lymphoma and grade $3 \mathrm{~b}$ follicular lymphoma patients responding to firstline treatment with rituximab plus cyclophosphamide, doxorubicin, vincristine, and prednisone: a prospective phase II clinical trial. Transl Cancer Res 2018;7:1044-53.

5. Tan L, Lin P, Chisti MM, et al. Real time analysis of binding between Rituximab (anti-CD20 antibody) and B lymphoma cells. Anal Chem 2013;85:8543-51.

6. Mian M, Marcheselli L, Rossi A, et al. A diachroniccomparative analysis for the identification of the most powerful prognostic index for localized diffuse large B-cell 
lymphoma. Ann Oncol 2014;25:2398-404.

7. Yin L, Xu-Monette ZY, Brock J, et al. Different prevalence and clinical outcome of Epstein-Barr virus positive diffuse large B-cell lymphoma between North American and nonWestern populations. Ann Transl Med 2018;6:236.

8. Crump M, Neelapu SS, Farooq U, et al. Outcomes in refractory diffuse large B-cell lymphoma: results from the international SCHOLAR-1 study. Blood 2017;130:1800-8.

9. Telio D, Fernandes K, Ma C, et al. Salvage chemotherapy and autologous stem cell transplant in primary refractory diffuse large B-cell lymphoma: outcomes and prognostic factors. Leuk Lymphoma 2012;53:836-41.

10. Christian Gisselbrecht, Bertram Glass, Nicolas Mounier, et al. Salvage regimens with autologous transplantation for relapsed large B-cell lymphoma in the rituximab era. J Clin Oncol 2010;28:4184-90.

11. Figueroa JA, Reidy A, Mirandola L, et al. Chimeric antigen receptor engineering: a right step in the evolution of adoptive celluIar immunotherapy. Int Rev Immunol 2015;34:154-87.

12. Kochenderfer JN, Rosenberg SA. Treating B-cell cancer with $\mathrm{T}$ cells expressing anti-CD19 chimeric antigen receptors. Nat Rev Clin Oncol 2013;10:267-76.

13. Enblad G, Karlsson H, Loskog AS. CAR T-Cell Therapy: The Role of Physical Barriers and Immunosuppression in Lymphoma. Hum Gene Ther 2015;26:498-505.

14. SS Neelapu, FL Locke, NL Bartlett, et al. Axicabtagene ciloleucel CAR T-cell therapy in refractory large B-cell lymphoma. N Engl J Med 2017;377:2531-44.

15. Stephen SJ, Bishop MR, Tam CS, et al. Primary analysis of Juliet: a global, pivotal, phase 2 trial of CTL019 in adult patients with relapsed or refractory diffuse large B-cell lymphoma. Blood 2017;130:577.

16. Abramson JS, Palomba ML, Gordon LI, et al. High durable $C R$ rates in relapsed/refractory $(R / R)$ aggressive B-NHL treated with the CD19-directed CAR T cell product JCAR017 (TRANSCEND NHL 001): defined composition allows for dose-finding and definition of pivotal cohort. Blood 2017;130:81.

17. Neelapu SS. An interim analysis of the ZUMA-1 study of KTE-C19 in refractory, aggressive non-Hodgkin lymphoma. Clin Adv Hematol Oncol 2017;15:117-20.

18. Locke FL, Neelapu SS, Bartlett NL, et al. Phase 1 Results of ZUMA-1: A Multicenter Study of KTE-C19 AntiCD19 CAR T Cell Therapy in Refractory Aggressive Lymphoma. Mol Ther 2017;25:285-95.

19. Schuster SJ, Bishop MR, Tam CS, et al. Tisagenlecleucel in Adult Relapsed or Refractory Diffuse Large B-Cell
Lymphoma. N Engl J Med 2019;380:45-56.

20. Chow VA, Shadman M, Gopal AK. Translating antiCD19 CAR T-cell therapy into clinical practice for relapsed/refractory diffuse large B-cell lymphoma. Blood 2018;132:777-81

21. Lee WL, Slutsky AS. Sepsis and endothelial permeability. N Engl J Med 2010;363:689-91.

22. Kochenderfer JN, Somerville RPT, Lu T, et al. Lymphoma Remissions Caused by Anti-CD19 Chimeric Antigen Receptor T Cells Are Associated With High Serum Interleukin-15 Levels. J Clin Oncol 2017;35:1803-13.

23. International Non-Hodgkin's Lymphoma Prognostic Factors Project. A predictive model for aggressive nonHodgkin's lymphoma. N Engl J Med 1993;329:987-94.

24. Hoster E, Dreyling M, Klapper W, et al. A new prognostic index (MIPI) for patients with advanced-stage mantle cell lymphoma. Blood 2008;111:558-65.

25. Futian Ma, Jin-Yuan Ho, Huan Du, et al. Evidence of long-lasting anti-CD19 activity of engrafted CD19 chimeric antigen receptor-modified $\mathrm{T}$ cells in a phase I study targeting pediatrics with acute lymphoblastic leukemia. Hematological Oncology 2019;37: 601-08.

26. Lee DW, Gardner R, Porter DL, et al. Current concepts in the diagnosis and management of cytokine release syndrome. Blood 2014;124:188-95.

27. Neelapu SS, Tummala S, Kebriaei P, et al. Chimeric antigen receptor T-cell therapy - assessment and management of toxicities. Nat Rev Clin Oncol 2018;15:47-62.

28. Fermé C, Mounier N, Casasnovas O, et al. Long-term results and competing risk analysis of the H89 trial in patients with advanced-stage Hodgkin lymphoma: a study by the Groupe d'Etude des Lymphomes de l'Adulte (GELA). Blood 2006;107:4636-42.

29. Thierry Philip, Cesare Guglielmi, Anton Hagenbeek, et al. Autologous bone marrow transplantation as compared with salvage chemotherapy in relapses of chemotherapysensitive non Hodgkin's lymphoma. N Engl J Med 1995;333:1540-45.

30. Vose JM, Zhang MJ, Rowlings PA, et al. Autologous transplantation for diffuse aggressive non-Hodgkin's lymphoma in patients never achieving remission: a report from the Autologous Blood and Marrow Transplant Registry. J Clin Oncol 2001;19:406-13.

31. Elstrom RL, Martin P, Ostrow K, et al. Response to second-line therapy defines the potential for cure in patients with recurrent diffuse large B-cell lymphoma: implications for the development of novel therapeutic strategies. Clin Lymphoma Myeloma Leuk 2010;10:192-6. 
32. Van Den Neste E, Schmitz N, Mounier N, et al. Outcomes of diffuse large B-cell lymphoma patients relapsing after autologous stem cell transplantation: an analysis of patients included in the CORAL study. Bone Marrow Transplant 2017;52:216-21.

33. Nagle SJ, Woo K, Schuster SJ, et al. Outcomes of patients with refractory/relapsed diffuse large B-cell lymphoma who progress after autologous stem cell transplantation in the rituximab era. Am J Hematol 2013;88:890-94.

34. Cheadle EJ, Gornall H, Baldan V, et al. CAR T cells: driving the road from the laboratory to the clinic. Immunol Rev 2014;257:91-106.

35. Dai H, Wang Y, Lu X, et al. Chimeric Antigen Receptors Modified T-Cells for Cancer Therapy. J Natl Cancer Inst 2016;108:djv439.

36. Jensen MC, Popplewell L, Cooper LJ, et al. Antitransgene rejection responses contribute to attenuated persistence of adoptively transferred CD20/CD19-specific chimeric antigen receptor redirected $\mathrm{T}$ cells in humans. Biol Blood Marrow Transplant 2010;16:1245-56.

37. Haso W, Lee DW, Shah NN, et al. Anti-CD22-chimeric antigen receptors targeting $\mathrm{B}$-cell precursor acute lymphoblastic leukemia. Blood 2013;121:1165-74.

38. Xiao X, Jiang YY, Cao YQ, et al. Efficacy and safety of CD19 chimeric antigen receptor $T$ cells for the treatment of 22 patients with B-cell lymphoma. Zhonghua Xue Ye Xue Za Zhi 2019;40:276-80.

39. Phan J, Mazloom A, Medeiros LJ, et al. Benefit of consolidative radiation therapy in patients with diffuse large B-cell lymphoma treated with R-CHOP chemotherapy. J Clin Oncol 2010;28:4170-6.

40. van Riggelen J, Yetil A, et al. MYC as a regulator of ribosome biogenesis and protein synthesis. Nat Rev Cancer 2010;10:301-9.

41. Eilers M, Eisenman RN. Myc's broad reach. Genes Dev 2008;22:2755-66.

42. Slack GW, Gascoyne RD. MYC and aggressive B-cell lymphomas. Adv Anat Pathol 2011;18:219-28.

43. Swerdlow SH, Campo E, Pileri SA, et al. The 2016 revision of the World Health Organization classification of lymphoid neoplasms. Blood 2016;127:2375-90.

44. Valera A, López-Guillermo A, Cardesa-Salzmann T, et al. MYC protein expression and genetic alterations have prognostic impact in patients with diffuse large B-cell lymphoma treated with immunochemotherapy. Haematologica 2013;98:1554-62.

45. Foot NJ, Dunn RG, Geoghegan H, et al. Fluorescence in situ hybridisation analysis of formalin-fixed paraffin- embedded tissue sections in the diagnostic work-up of non-Burkitt high grade B-cell non-Hodgkin's lymphoma: a single centre's experience. J Clin Pathol 2011;64:802-8.

46. Gupta M, Maurer MJ, Wellik LE, et al. Expression of Myc, but not pSTAT3, is an adverse prognostic factor for diffuse large B-cell lymphoma treated with epratuzumab/ R-CHOP. Blood2012;120:4400-6.

47. Fry TJ, Shah NN, Orentas RJ, et al. CD22-targeted CAR T cells induce remission in B-ALL that is naive or resistant to CD19-targeted CAR immunotherapy. Nat Med 2018;24:20-8.

48. Ruella M, Xu J, Barrett DM, et al. Induction of resistance to chimeric antigen receptor $\mathrm{T}$ cell therapy by transduction of a single leukemic B cell. Nat Med 2018;24:1499-503.

49. Ruella M, Barrett DM, Kenderian SS, et al. Dual CD19 and CD123 targeting prevents antigen-loss relapses after CD19-directed immunotherapies. J Clin Invest 2016;126:3814-26.

50. Larouche JF, Berger F, Chassagne-Clément C, et al. Lymphoma recurrence 5 years or later following diffuse large B-cell lymphoma: clinical characteristics and outcome. J Clin Oncol 2010;28:2094-100.

51. Advani R, Flinn I, Popplewell L, et al. CD47 Blockade by Hu5F9-G4 and Rituximab in Non-Hodgkin's Lymphoma. N Engl J Med 2018;379:1711-21.

52. Flowers CR, Sinha R, Vose JM. Improving outcomes for patients with diffuse large B-cell lymphoma. CA Cancer J Clin 2010;60:393-408.

53. Norelli M, Camisa B, Barbiera G, et al. Monocyte-derived IL-1 and IL-6 are differentially required for cytokinerelease syndrome and neurotoxicity due to CAR T cells. Nat Med 2018;24:739-48.

54. Pennell CA, Barnum JL, McDonald-Hyman CS, et al. Human CD19-Targeted Mouse T Cells Induce B Cell Aplasia and Toxicity in Human CD19 Transgenic Mice. Mol Ther 2018;26:1423-34.

55. Liu D, Zhao J. Cytokine release syndrome: grading, modeling, and new therapy. J Hematol Oncol 2018;11:121.

56. Abbasi J. Safer CAR T-Cell Therapy. JAMA 2019;321:2155.

57. Minton K. Immunotherapy: Cytokine boost for CAR T cells. Nat Rev Immunol 2018;18:150-1.

Cite this article as: Huang C, Wu L, Liu R, Li W, Li Z, Li J, Liu L, Shan B. Efficacy and safety of CD19 chimeric antigen receptor $\mathrm{T}$ cells in the treatment of 11 patients with relapsed/ refractory B-cell lymphoma: a single-center study. Ann Transl Med 2020;8(17):1048. doi: 10.21037/atm-20-4363 


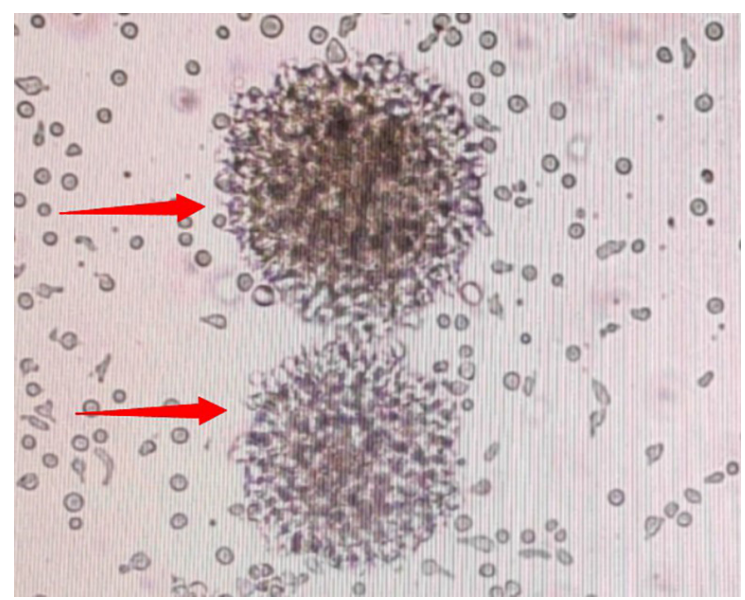

Figure S1 A representative image of anti-CD19 CAR-T cells. 